\title{
Efecto de las condiciones de enfriamiento sobre la fracción y morfología de la fase primaria épsilon en una aleación comercial ACuZinc5 ${ }^{\mathrm{TM}(\cdot)}$
}

\author{
E.D. Jareño*, S.I. Maldonado* y F.A. Hernández*
}

Resumen

\begin{abstract}
La fracción sólida de la fase primaria épsilon $(\varepsilon)$ y su interrelación con la fase eta $(\eta)$ influyen en las propiedades mecánicas de la aleación ACuZinc5TM ${ }^{\mathrm{TM}}$. En este estudio se midió la fracción de fase épsilon primaria resultante de condiciones de enfriamiento similares a las que se utilizan en diferentes procesos de vaciado. Adicionalmente se realizó un análisis a partir de los cambios morfológicos de la fase primaria y de los resultados del análisis térmico para sugerir el momento probablemente adecuado para agitar el baño metálico durante procesos de vaciado en estado semisólido. A partir de estos resultados es posible diseñar ciclos térmicos experimentales para obtener una apropiada distribución de las fases $\varepsilon$ y $\eta$ en la microestructura final de las piezas vaciadas.
\end{abstract}

Palabras Clave Fase épsilon; Análisis térmico; Condiciones de enfriamiento; Aleación ACuZinc5 ${ }^{\mathrm{TM}}$.

\section{The cooling conditions effects on the fraction and morphology of the epsilon primary phase in an ACuZinc $5^{\mathrm{TM}}$ commercial alloy}

\begin{abstract}
The mechanical properties of ACuZinc $5^{\mathrm{TM}}$ alloys are influenced by the fraction solid of the primary epsilon $(\varepsilon)$ phase and their interrelations with the eta $(\eta)$ phase. In this study the primary epsilon phase fraction results from cooling conditions similar to that applied in different casting processes have been measured. Additionally an analysis was made from the morphology's changes and from the thermal analysis results to suggest the probably adequate moment to apply stirring in semisolid metal processing. From these results it is possible to design experimental thermal cycles to obtain an adequate distribution of $\varepsilon$ and $\eta$ phases in the end castings microstructure.
\end{abstract}

Keywords

Phase epsilon; Thermal analysis; Cooling conditions; ACuZinc5 $5^{\mathrm{TM}}$ alloys.

\section{INTRODUCCIÓN}

De las aleaciones de zinc del sistema $\mathrm{Zn}$-Al-Cu que se utilizan en vaciado en dados a alta presión (HPDC) con la que se obtienen mejores propiedades mecánicas es con la aleación ACuZinc5 $5^{\mathrm{TM}[1}$ y 2]. Estos resultados dependen de la distribución e interrelación entre las fases épsilon $\left(\varepsilon, \mathrm{Zn}_{4} \mathrm{Cu}\right)$ y eta ( $\eta$, solución sólida rica en zinc). La microestructura final de esta aleación vaciada por HPDC se caracteriza por una combinación íntima de rosetas finas de fase primaria $\varepsilon$ como substrato de nucleación de un peritéctico de fase $\eta$ en una matriz eutéctica ternaria $^{[1}$ y 2$]$.

El HPDC consiste en inyectar metal fundido a alta presión en un molde metálico enfriado. Las altas velocidades de enfriamiento, la solidificación de cristales en el sistema de inyección (fuera del molde) y la dinámica del flujo al llenar el molde influyen en el tamaño, distribución y morfología de las fases solidificadas ${ }^{[3]}$.

Utilizando el HPDC en cámara caliente se logran producir piezas precisas con una alta productividad, pero se forman defectos tales como porosidad y una microestructura no uniforme. En contraste, dos de las ventajas de los procesos de vaciado en estado semisólido (SSM) son la reducción de la porosidad y la obtención de una microestructura uniforme ${ }^{[4]}$. En los procesos SSM la aleación metálica fundida no se vacía en el molde directamente desde el líquido. Antes de vaciar, la aleación líquida permanece isotérmicamente o se enfría lentamente en el rango de

(•) Trabajo recibido el día 24 de octubre de 2011 y aceptado en su forma final el día 26 de mayo de 2012

* Universidad Autónoma de Zacatecas. Av. López Velarde 801, 98060 Zacatecas, México, Tel-fax: +52(492)9239407, +52(492)9220827. 
crecimiento (engrosamiento o maduración) de la fase primaria. Durante este procedimiento se modifica la morfología de la fase primaria mediante agitación mecánica, vibraciones, manipulando las condiciones de nucleación y crecimiento, etc. ${ }^{[5}$ y] . $^{6}$

En ambos procesos de vaciado, HPDC y SSM, el resultado final está relacionado con la fracción de fase primaria formada ${ }^{[1,2 \text { y } 6]}$. La fracción de fase $\varepsilon$ primaria en estas aleaciones ha sido medida en trabajos previos pero en condiciones de enfriamiento y composición química diferentes ${ }^{[7]}$. Debido a la relación directa de las condiciones de enfriamiento con la microestructura final, en el presente artículo se compara el efecto de condiciones de enfriamiento similares a las utilizadas en procesos HPDC, SSM y vaciado por gravedad en la fracción y morfología de la fase primaria $\varepsilon$ en una aleación ACuZinc $5^{\mathrm{TM}}$.

\section{PROCEDIMIENTO EXPERIMENTAL}

La aleación ACuZinc5 $5^{\mathrm{TM}}$ utilizada fue adquirida del fabricante autorizado Eastern Alloys. Su composición química está dentro de la norma ASTM B892 para este tipo de aleaciones de zinc, fue verificada antes y después de la experimentación mediante espectrometría de absorción atómica. Contenido de aleantes: 5,58 Cu; 3,18 Al; 0,04 Mg.

\subsection{Análisis térmico}

Las temperaturas de reacción y la del punto de coherencia dendrítica se determinaron mediante la técnica de análisis térmico por curvas de enfriamiento. Las temperaturas de reacción fueron calculadas por medio de la técnica de la derivada utilizada por Bäckerud y colaboradores $^{[8 \mathrm{y} 9]}$ y el punto de coherencia dendrítica (PCD) se determinó mediante la técnica de la diferencia de temperaturas utilizada al efecto por Arnberg,

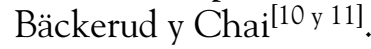

Para describir las técnicas aplicadas en la figura 1 se muestran las curvas de enfriamiento, derivadas y diferencia de temperaturas obtenidas en el presente estudio con una de las muestras ensayadas.

La reacción primaria (IRP) inicia en la periferia de la muestra y se determina proyectando el punto donde cambia abruptamente de dirección la curva $\mathrm{dT} / \mathrm{dt}$ sobre la curva de enfriamiento Tp y de este al eje de las temperaturas. De manera similar se determina gráficamente el fin de la reacción primaria (FRP), en este caso se considera que la reacción de formación de fase primaria desde el líquido termina en el centro de la muestra. Se utiliza la intercepción de la recta " $a$ " tangente a la curva dTc/dt y la recta promedio " $b$ " para determinar el punto de inflexión, de este a la curva Tc y de ahí al eje de las temperaturas. El PCD se estima proyectando sobre la curva Tc el punto donde la curva de diferencia de temperaturas $\mathrm{Tp}$ - Tc alcanza su valor mínimo promedio después de la primera reacción ${ }^{[11]}$, en la figura 1 , se proyecta el punto " $i$ ", a la curva Tc y de ahí al eje de las temperaturas.

Para aplicar estas técnicas se colocaron dos termopares tipo "K", uno en el centro y otro en la pared a la mitad de la altura interior de un molde de grafito de $40 \mathrm{~mm}$ de diámetro interior, $46 \mathrm{~mm}$ de diámetro exterior, $47 \mathrm{~mm}$ de altura exterior y $3 \mathrm{~mm}$ de espesor del fondo. Tanto el fondo como la tapa fueron aislados térmicamente para que la extracción de calor fuera a través de las paredes del molde. Con esta configuración se obtuvieron velocidades de enfriamiento entre $0,3^{\circ} \mathrm{C} / \mathrm{s}$ y $0,5^{\circ} \mathrm{C} / \mathrm{s}$ (enfriamiento natural) y $0,9^{\circ} \mathrm{C} / \mathrm{s}$ (aire forzado).

Para obtener velocidades de enfriamiento próximas a $18^{\circ} \mathrm{C} / \mathrm{s}$ se utilizó un termopar en el centro de la muestra en el molde descrito en la figura 2 a). En este caso, mediante el termopar inserto en el cuerpo del crisol, se verificó que la temperatura del mismo alcanzó los $240 \pm 5^{\circ} \mathrm{C}$, mientras que la temperatura del metal líquido al vaciarlo varió a los $550 \pm 5^{\circ} \mathrm{C}$.

Con la velocidad de enfriamiento de $18^{\circ} \mathrm{C} / \mathrm{s}$ se obtuvieron tiempos de solidificación inferiores a los $8 \mathrm{~s}$ reportados en la fabricación de piezas de aleaciones de cinc mediante procesos HPDC [12].

La velocidad de enfriamiento de $0,4^{\circ} \mathrm{C} / \mathrm{s}$ se utilizó para determinar las temperaturas de reacción a partir de las cuales se diseñaron los ciclos térmicos descritos en el apartado 2,2 y para medir el efecto de esta velocidad sobre la fase primaria. Las demás velocidades de enfriamiento $\left(0,9^{\circ} \mathrm{C} / \mathrm{s}\right.$ y $\left.18^{\circ} \mathrm{C} / \mathrm{s}\right)$ sólo se utilizaron para medir su efecto sobre la fracción y morfología de la fase $\varepsilon$.

La precisión del dispositivo de adquisición de datos ${ }^{[13]}$ fue verificada obteniendo las curvas de enfriamiento de zinc con 99,99\% de pureza. Las mediciones realizadas fueron comprobadas utilizando un calibrador marca Omega modelo CL27.

\subsection{Condiciones de enfriamiento utilizadas}

Para medir el efecto sobre la microestructura al experimentar diferentes condiciones de enfriamiento se empleó la técnica de templar desde las temperaturas de interés. Se utilizaron las tempera- 


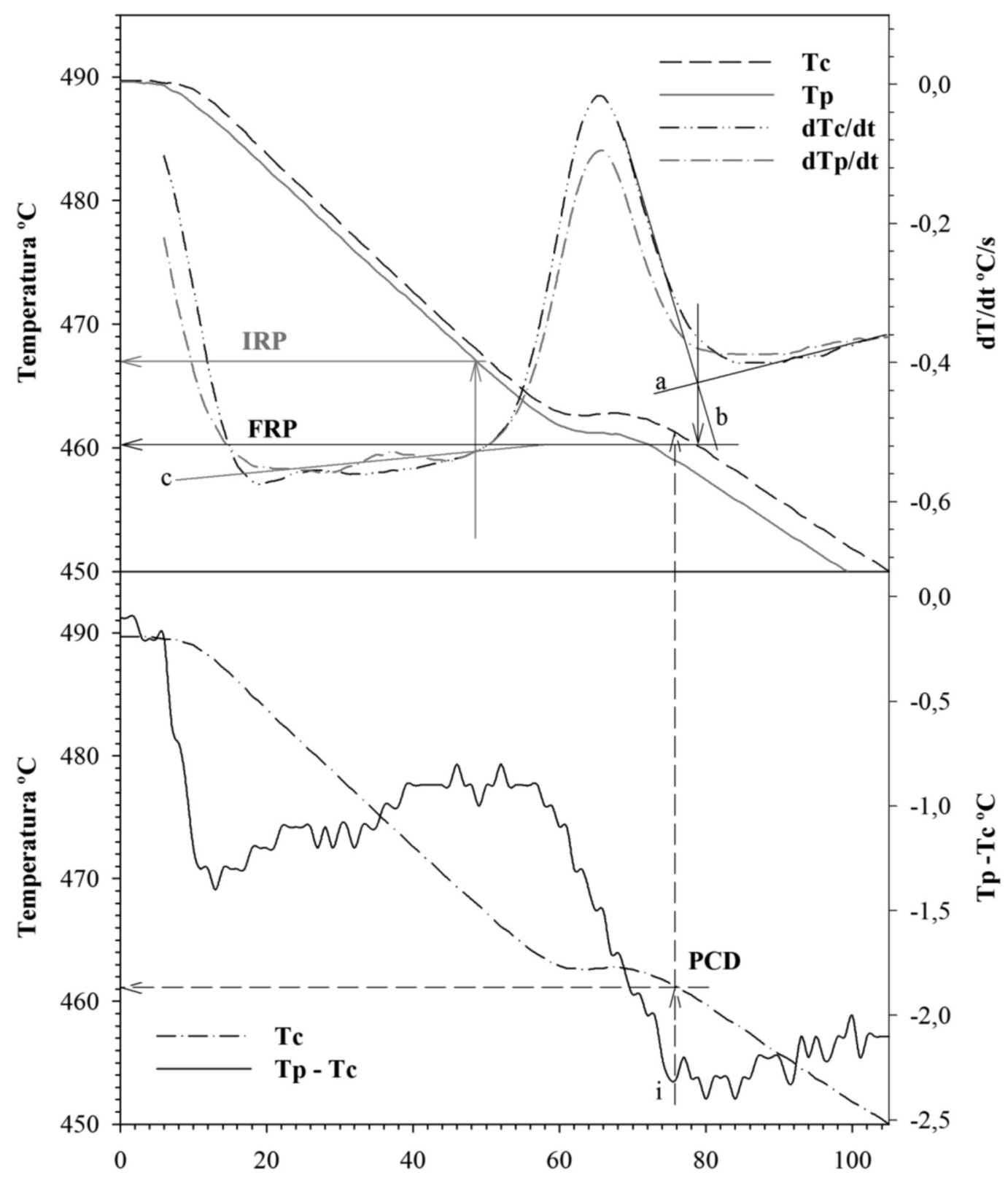

Tiempo s

Figura 1. Aleación ACuZinc5 $5^{\mathrm{TM}}$. Fragmento de la curva de enfriamiento correspondiente a la reacción primaria mostrada como reacción 1 en la figura 3. Parte superior, técnica de la derivada. Parte inferior, técnica de la diferencia de temperaturas. Inicio de la reacción primaria (IRP), fin de la reacción primaria (FRP). Punto de coherencia dendrítica (PCD) Tc, temperatura en el centro, Tp temperatura en la periferia. Velocidad de enfriamiento: $0,4{ }^{\circ} \mathrm{C} / \mathrm{s}$.

Figure 1. ACuZinc5 ${ }^{T M}$ alloy. Cooling curve fragment corresponding to the primary reaction shown as reaction 1 on figure 3. Top, derivative technique. Bottom, difference of temperature technique. Primary reaction beginning (IRP), primary reaction ending (FRP). Dendrite coherency point (PCD), Tc, center temperature. Tp, edge temperature. Cooling rate: $0.4^{\circ} \mathrm{C} / \mathrm{s}$.

turas de reacción determinadas en este estudio. El temple se efectuó en agua a aproximadamente $7^{\circ} \mathrm{C}$.
En todos los casos se templaron tres muestras. Se colocó un termopar tipo K en el centro del molde 


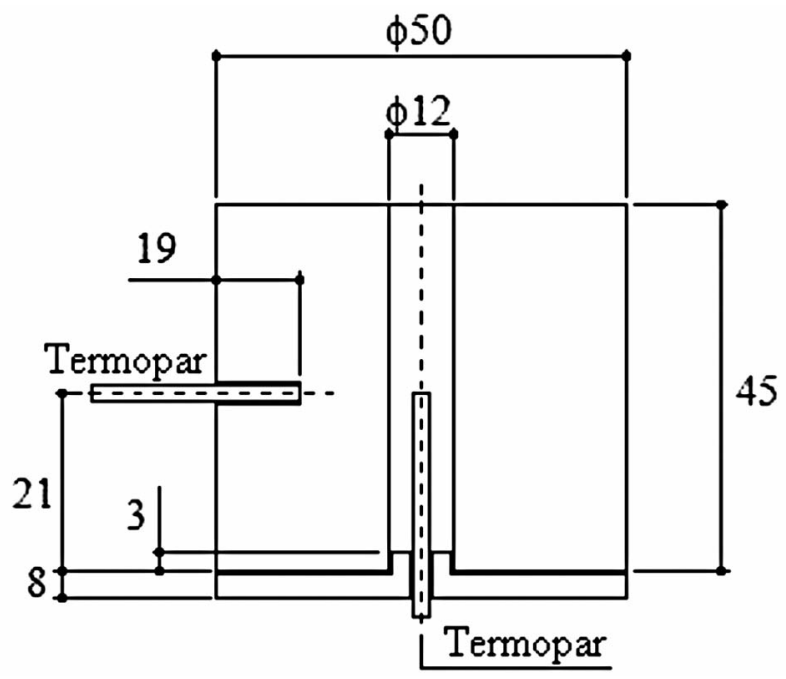

Figura 2 a). Copa de grafito para velocidades de enfriamiento de $18^{\circ} \mathrm{C} / \mathrm{s}$.

Figure 2 a). Graphite cup for $18^{\circ} \mathrm{C} / \mathrm{s}$ of cooling rate.

descrito en la figura $2 \mathrm{~b}$ ) y se sobrecalentaron las muestras hasta $490^{\circ} \mathrm{C}$ enfriándolas a $0,4^{\circ} \mathrm{C} / \mathrm{s}$ hasta iniciar las condiciones de enfriamiento descritas a continuación:
Tubo de Alúmina con Termopar

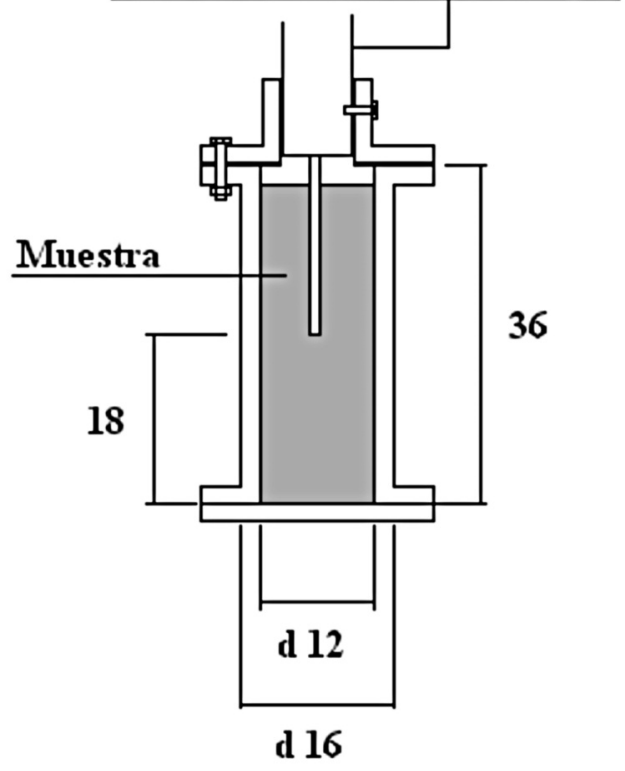

Figura 2 b). Copa de grafito para templar.

Figure 2 b). Graphite cup to quench.

- CI) Enfriamiento a altas velocidades desde líquidus. Con esta condición se busca medir

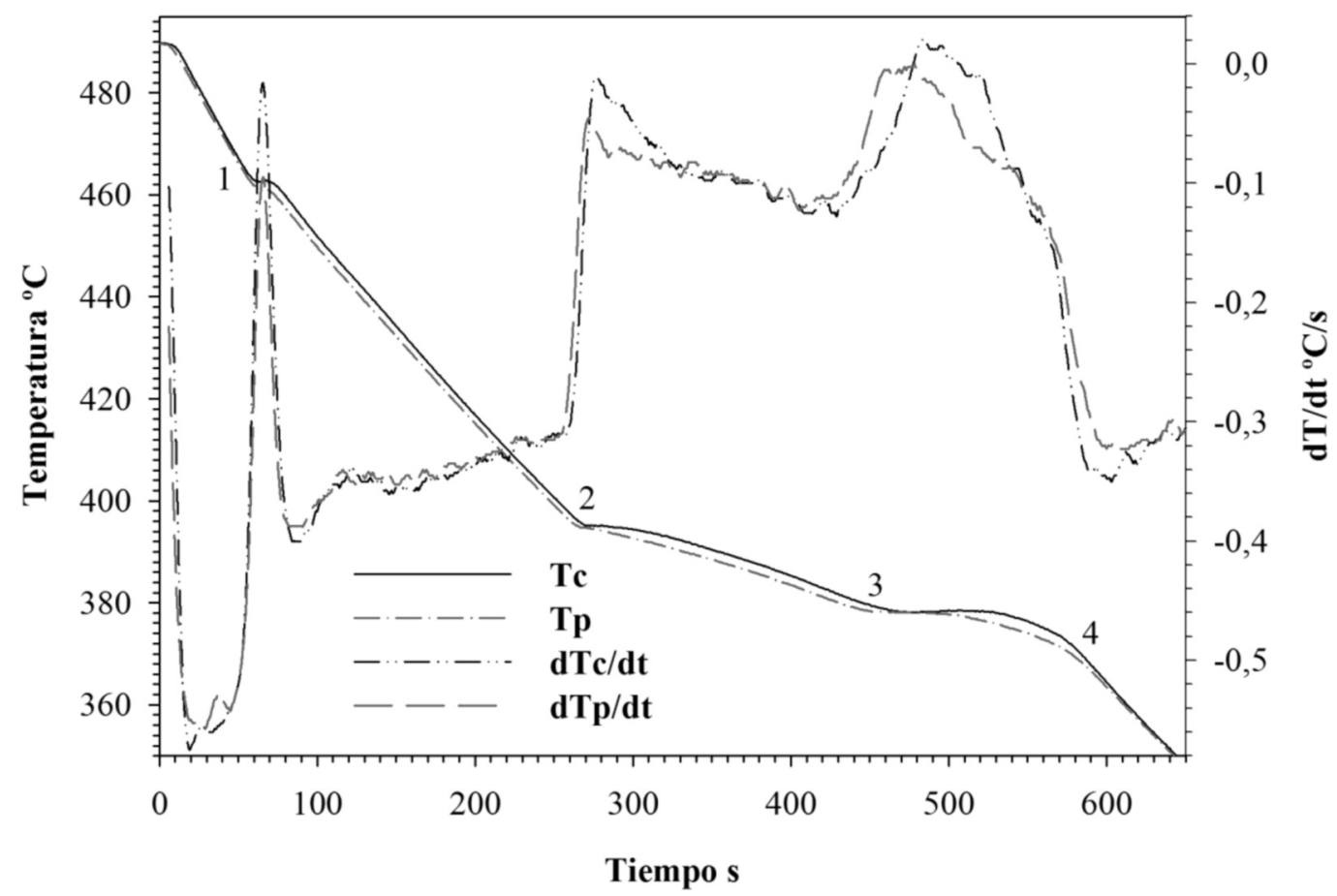

Figura 3. Aleación ACuZinc $5^{\mathrm{TM}}$. Curvas de enfriamiento y derivadas a $0,4{ }^{\circ} \mathrm{C} / \mathrm{s}$. 1- Reacción primaria, 2 - Formación del peritéctico de fase $\eta, 3$ - Formación del eutéctico ternario, 4 - Fin de la solidificación.

Figure 3. ACuZinc $5^{T M}$ alloy. Cooling and derivative curves at $0.4{ }^{\circ} \mathrm{C} / \mathrm{s} .1$ - Primary reaction. 2 - Formation of $\eta$ phase peritectic. 3- Formation of ternary eutectic. 4 - End of solidification. 
exclusivamente la fracción de fase primaria en ese punto y observar la microestructura antes del engrosamiento dendrítico. Se templaron las muestras desde la temperatura promedio de inicio de la reacción primaria determinada en el presente estudio.

- CII) Temple después de $1 \mathrm{~h}$ de permanencia a la temperatura de fin de la reacción primaria. En este caso se mide el efecto de la permanencia y enfriamiento brusco posterior sobre la fracción, tamaño y morfología de la fase primaria. No se agita la muestra con el objetivo de observar la evolución de la fase primaria en estas condiciones y estimar, en comparación con la microestructura obtenida con la condición I, en qué momento podría ser apropiado iniciar la agitación del baño metálico en caso de aplicar un proceso SSM.

- CIII) Enfriamiento desde el líquido a velocidades de enfriamiento de 0,$4 ; 0,9$ y $18{ }^{\circ} \mathrm{C} / \mathrm{s}$. Se aplicó para determinar la tendencia del efecto de la velocidad de enfriamiento sobre la fracción, tamaño y distribución de la fase primaria en este rango de velocidades de enfriamiento.

\subsection{Análisis microestructural. Análisis de imágenes}

Las fases $\varepsilon$ y $\eta$ fueron identificadas en trabajos previos $^{[13]}$. La fracción de fase primaria fue medida por análisis de imágenes de las muestras obtenidas por análisis térmico y por temple. Las muestras fueron seccionadas transversal y longitudinalmente. Las secciones transversales se realizaron en el plano adya- cente inferior de la punta de los termopares, y las longitudinales, en un plano paralelo a los termopares. Se midió la composición química en las dos direcciones mediante un espectrómetro de dispersión de energía (EDS) acoplado a un microscopio electrónico de barrido Jeol JSM-6300.

La preparación metalográfica de las muestras se inició desbastando con lijas desde grano 100 a 1.200 enfriando con agua a $240 \mathrm{rpm}$. El pulido se realizó en dos etapas, primero con una suspensión de alúmina de $0,3 \mu \mathrm{m}$ en agua destilada a $240 \mathrm{rpm}$ y finalmente a mano con una suspensión de sílice coloidal de 0,05 $\mu \mathrm{m}$ en agua destilada. El ataque químico usado fue el reactivo Tinofeef ${ }^{[14]}$. El análisis de imágenes se efectuó en un microscopio óptico Olympus Vanox AHMT3 con una cámara digital Olympus DP11 conectada a una PC con el software Image-Pro Plus 4.0. La cuantificación de fase $\varepsilon$ se obtuvo midiendo 20 campos a 200x.

\section{RESULTADOS}

\subsection{Análisis térmico}

El resultado de la medición de las temperaturas de reacción y del punto de coherencia dendrítica mediante las curvas de enfriamiento, derivadas y diferencia de temperaturas se resumen en la tabla I.

En la figura 1 se muestra la aplicación de estas técnicas. En esta figura y en los resultados resumidos en la tabla I se observa que el fin de la reacción primaria (FRP) y el punto de coherencia dendrítica (PCD) determinado por estas técnicas ocurren en un intervalo estrecho de temperaturas. Este puede ser considerado mínimo en relación al rango de temperaturas de crecimiento (engrosamiento) de la fase

Tabla I. Resultados del análisis térmico.

$\mathrm{T}_{\text {SC }}$, temperatura de sobrecalentamiento.

IRP, inicio de la reacción primaria. FRP, fin de la reacción primaria. $\mathrm{PCD}$, punto de coherencia dendrítica

Table I. Thermal analysis results. $T_{S C}$, superheat temperature. IRP, primary reaction beginning.

FRP, primary reaction ending. $P C D$, dendritic coherency point

\begin{tabular}{ccccc}
\hline \# Muestra & $\mathbf{T}_{\mathbf{S C}}{ }^{\circ} \mathbf{C}$ & IRP $^{\circ} \mathbf{C}$ & FRP $^{\circ} \mathbf{C}$ & PCD $^{\circ} \mathbf{C}$ \\
\hline 1 & 490,2 & 465,1 & 462,3 & 460,7 \\
2 & 489,7 & 466,3 & 462,6 & 461,2 \\
3 & 489,8 & 467,0 & 460,2 & 461,1 \\
Promedio & 489,9 & 466,1 & 461,7 & 461,0 \\
\hline
\end{tabular}


primaria entre el fin de la reacción 1 y la reacción 2 (Figs. 1 y 3). Adicionalmente, la similitud entre los resultados obtenidos mediante las técnicas de la derivada y la diferencia de temperaturas para FRP y el $\mathrm{PCD}$, sugieren que a partir de estas temperaturas se inicia el engrosamiento de la fase primaria y la formación de una red sólida continua dentro del baño metálico con el consecuente incremento súbito de la resistencia al corte ${ }^{[3 \text { y } 10]}$. Por lo tanto, como las temperaturas del fin de la reacción primaria (FRP) y el PCD oscilan entre 460 y $463^{\circ} \mathrm{C}$ (Tabla I) y teniendo en cuenta que se calibraron errores de $\pm 1{ }^{\circ} \mathrm{C}$ al verificar las mediciones, se asumieron estas temperaturas como límites del rango de experimentación para aplicar la condición de enfriamiento II.

\subsection{Análisis microestructural}

En la figura 4 se muestran micrografías comparando el tamaño, morfología y distribución de la fase primaria $\varepsilon$ después de ser aplicadas las condiciones de enfriamiento I, II y III respectivamente. En la figura 4 a) se observa una distribución homogénea de dendritas finas de fase $\varepsilon$ primaria formadas directamente desde el líquido $\left(466^{\circ} \mathrm{C}\right)$.

Se obtiene una microestructura similar templando a $462^{\circ} \mathrm{C}$. En la figura 4 b) se muestra la microestructura formada después de una hora de permanencia a $461^{\circ} \mathrm{C}$.
Esta microestructura se caracteriza por una distribución no homogénea de dendritas de fase $\varepsilon$ primaria: dendritas de mayor tamaño rodeadas de dendritas finas de la misma fase. Estas dendritas finas son similares en morfología, tamaño y distribución a las expuestas en la figura 4 a).

En las figuras 4 c) y 4 d) se aprecia que al incrementar la velocidad de enfriamiento de 0,9 a $18^{\circ} \mathrm{C} / \mathrm{s}$ disminuye el tamaño de la fase $\varepsilon$ y se verifica la tendencia observada en la figura $4 \mathrm{~b}$ ) de un crecimiento y distribución no uniformes de la fase $\varepsilon$.

El microanálisis químico no indica que se produzca macrosegregación.

\subsection{Análisis de imágenes}

La tabla II resume los resultados de las mediciones promedio de la fracción de fase $\varepsilon$ primaria obtenidos a partir de las condiciones de enfriamiento planteadas, CI $\left(35^{\circ} \mathrm{C} / \mathrm{s}\right)$, CII temple después de $1 \mathrm{~h}$ de permanencia, $\mathrm{CIII}\left(0,4^{\circ} \mathrm{C} / \mathrm{s} ; 0,9^{\circ} \mathrm{C} / \mathrm{s}\right.$ y $\left.18^{\circ} \mathrm{C} / \mathrm{s}\right)$.

\section{DISCUSIÓN}

Con base en la tabla II, para estas condiciones de enfriamiento, la fracción de fase $\varepsilon$ primaria tiene la tendencia a disminuir con el incremento de la velo-

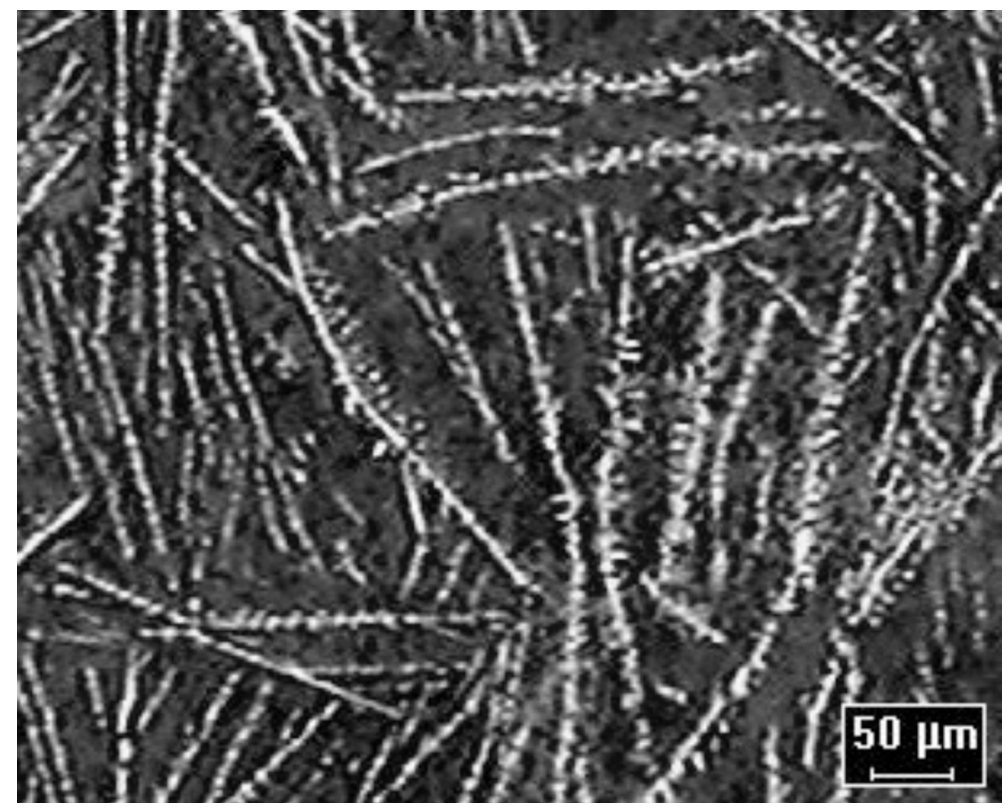

Figura 4 a). Aleación ACuZinc5 templada a $466{ }^{\circ} \mathrm{C}$ sin permanencia. Dendritas finas de fase $\varepsilon$ primaria.

Figure 4 a). ACuZinc5 alloy quenched at $466^{\circ} \mathrm{C}$ without permanency. Fine primary $\varepsilon$ phase dendrites. 


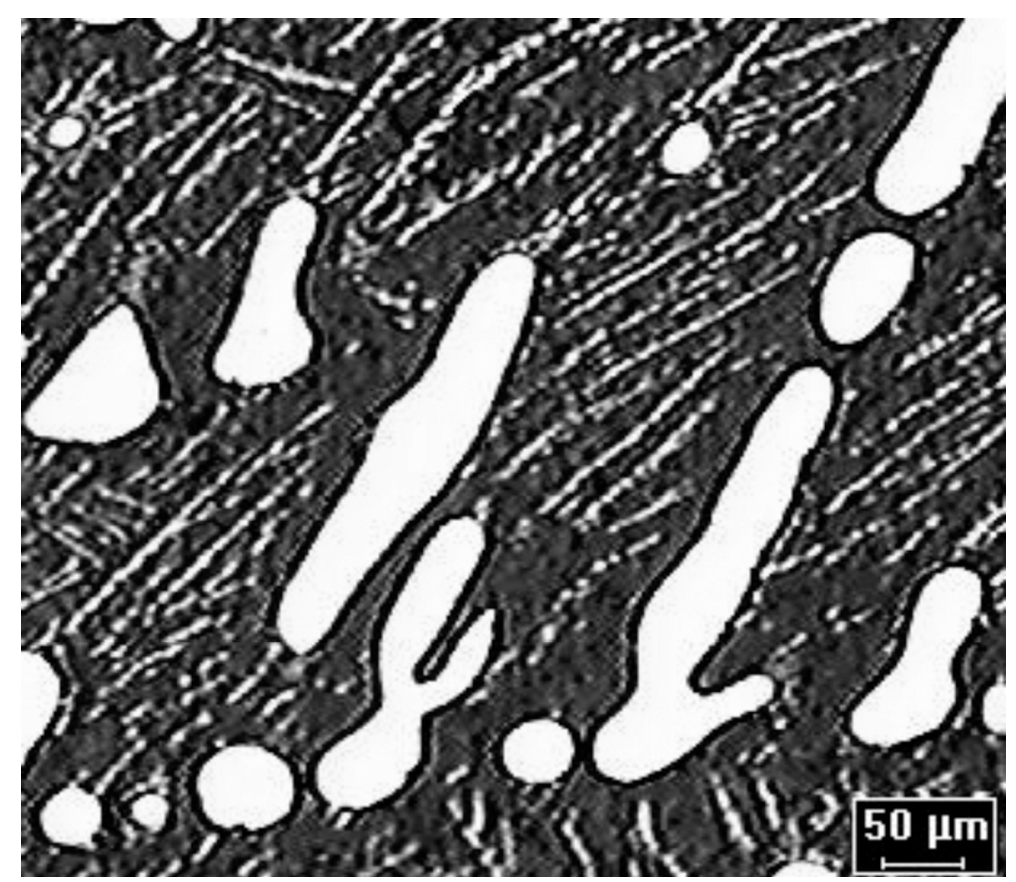

Figura 4 b). Aleación ACuZinc5 templada después de $1 \mathrm{~h}$ de permanencia a $461^{\circ} \mathrm{C}$. Dendritas finas y gruesas de fase $\varepsilon$ primaria.

Figure $4 \mathrm{~b}$ ). ACuZinc5 alloy quenched after $1 \mathrm{~h}$ of permanency at $461{ }^{\circ} \mathrm{C}$. Fine and larger primary $\varepsilon$ phase dendrites.

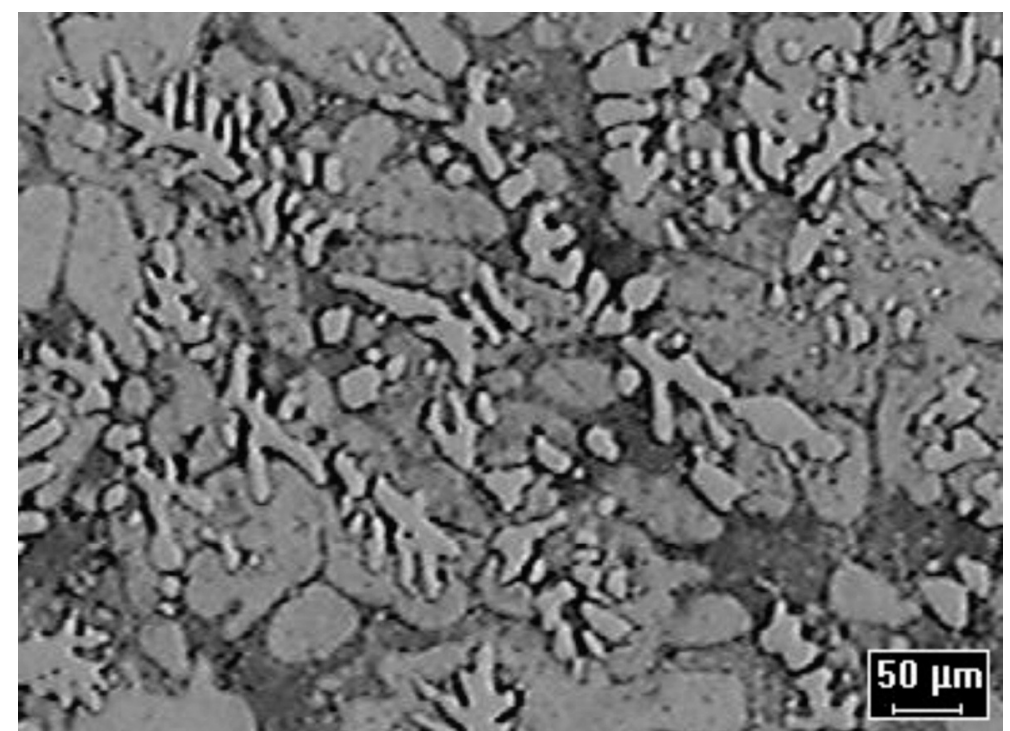

Figura 4 c). Aleación ACuZinc5. Velocidad de enfriamiento $0,9^{\circ} \mathrm{C} / \mathrm{s} .50 \mathrm{x}$

Figure $4 \mathrm{c}$ ). ACuZinc5 alloy. Cooling rate $0.9^{\circ} \mathrm{C} / \mathrm{s} .50 \mathrm{x}$

cidad de enfriamiento. Lo anterior está relacionado entre otros factores con la disminución de la solubilidad del aluminio y del cobre en zinc ${ }^{[15]}$ con la disminución de la temperatura, con las transformaciones de fase en estado sólido durante el enfriamiento continuo desde el líquido ${ }^{[15-18]}$ y con la disminución de los tiempos de solidificación local de $\varepsilon$ al incrementar la velocidad de enfriamiento ${ }^{[13]}$. En la aleación 


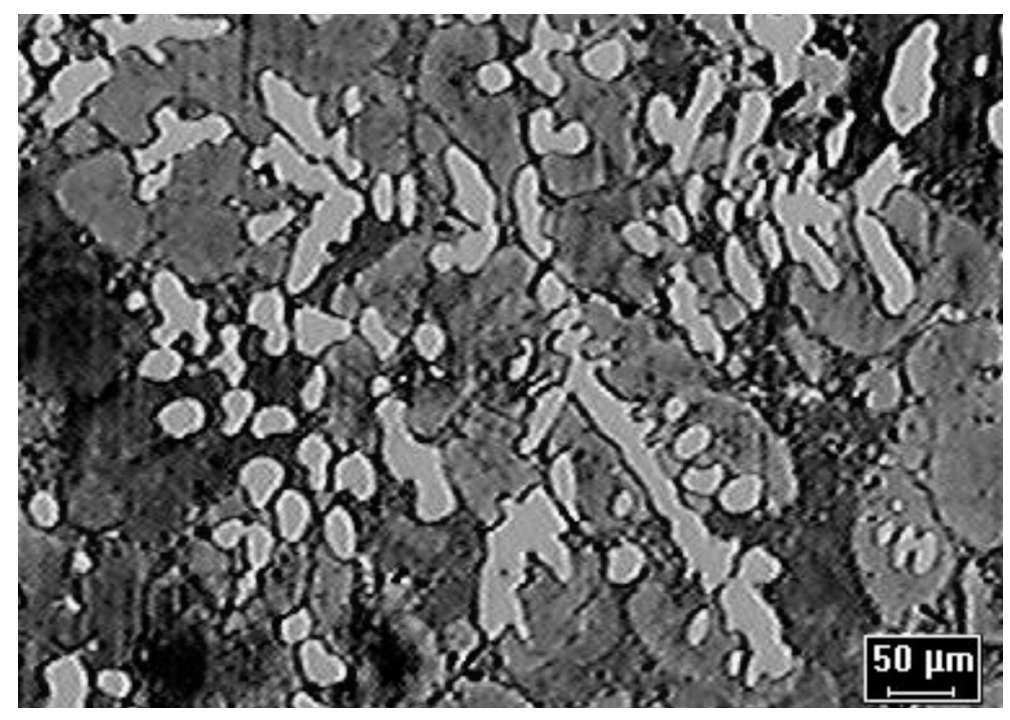

Figura 4 d). Aleación ACuZinc5. Velocidad de enfriamiento $0,4^{\circ} \mathrm{C} / \mathrm{s} .50 \mathrm{x}$

Figure 4 d). ACuZinc5 alloy. Cooling rate $0.4^{\circ} \mathrm{C} / \mathrm{s}$. 50x.

Tabla II. Efecto de la condición y de la velocidad de enfriamiento $\left({ }^{\circ} \mathrm{C} / \mathrm{s}\right)$ sobre la fracción de fase $\varepsilon$ primaria $\left(f_{s} \varepsilon\right)$. Cl, Cll y CIII

(Condiciones de enfriamiento I, II y III)

Table II. Effects of the condition and cooling rate $\left({ }^{\circ} \mathrm{C} / \mathrm{s}\right)$ on the primary $\varepsilon$ phase fraction $\left(f_{s} \varepsilon\right)$. Cl, Cll y CIII (Cooling conditions I, II y III)

\begin{tabular}{cccccc}
\hline & $\mathrm{Cl}\left(\sim 35^{\circ} \mathrm{C} / \mathrm{s}\right)$ & $\mathrm{CII}\left(1 \mathrm{~h} \mathrm{a} 461^{\circ} \mathrm{C}\right)$ & $\mathrm{CIII}\left(\mathbf{0 , 4}{ }^{\circ} \mathrm{C} / \mathrm{s}\right)$ & $\mathrm{CIII}\left(\mathbf{1}^{\circ} \mathrm{C} / \mathrm{s}\right)$ & $\mathrm{CIII}\left(18^{\circ} \mathrm{C} / \mathrm{s}\right)$ \\
\hline $\mathrm{f}_{\mathrm{s}} \varepsilon$ & 0,16 & 0,29 & 0,27 & 0,26 & 0,19 \\
\hline
\end{tabular}

ACuZinc5 al incrementar la velocidad de enfriamiento de 0,4 a $0,9^{\circ} \mathrm{C} / \mathrm{s}$ se reduce aproximadamente en un $50 \%$ el tiempo de solidificación local de $\varepsilon$ primaria $^{[13]}$.

Pudiera considerarse el efecto de la formación del peritéctico de fase $\eta$ en la disminución del crecimiento de $\varepsilon$ primaria, tal y como se describe que sucede en las aleaciones donde se produce reacción y transformación peritécticas ${ }^{[19}$ y 20$]$, pero de acuerdo con nuestros resultados en estas aleaciones no se completa la transformación peritéctica. En las micrografías a $1.000 \mathrm{x}$ de las muestras ensayadas a $18^{\circ} \mathrm{C} / \mathrm{s}$ se alcanza a observar que entre $\varepsilon$ primaria y $\eta$ se forma eutéctico ( Fig. 5), es decir, se produce una reacción peritéctica que no limita por encapsulamiento (transformación) el crecimiento de la fase properitéctica ${ }^{[20]}$, por lo que el crecimiento de $\varepsilon$ e incluso la formación y el tipo de reacción peritéctica pueden estar sujetos fundamentalmente a limitaciones difusivas asociadas a las condiciones de enfriamiento, tal y como se asume en una publicación consultada ${ }^{[7]}$ donde se estudió por medio de la técnica de Bridgman la reacción peritéctica en la aleación binaria $\mathrm{Zn}-\mathrm{Cu}$.

Al templar la muestra después de permanecer a la temperatura FRP (CII) se obtiene una mayor fracción de fase $\varepsilon$ primaria, pero con una distribución y tamaño no uniformes ( Fig. 4 b)). En esta figura se observa que el ancho de las dendritas de $\varepsilon$ más grandes es menor a los $50 \mu \mathrm{m}$ de tamaño en promedio de las rosetas de fase $\varepsilon$ observadas en la microestructura de una aleación ACuZinc vaciada por HPDC [1]. Las rosetas obtenidas por HPDC ${ }^{[1]}$ no son uniformes a pesar de que se inyecta el metal líquido directamente en el molde enfriado, y por tanto, según el resultado de la condición de enfriamiento I, se parte de una microestructura uniforme ( Fig. 4 a)). Este resultado apunta a que, efectivamente, la falta de uniformidad en la microestructura obtenida por HPDC se debe fundamentalmente a las variables implícitas al llenado del molde según se indica en trabajos anteriores ${ }^{[3]}$. 


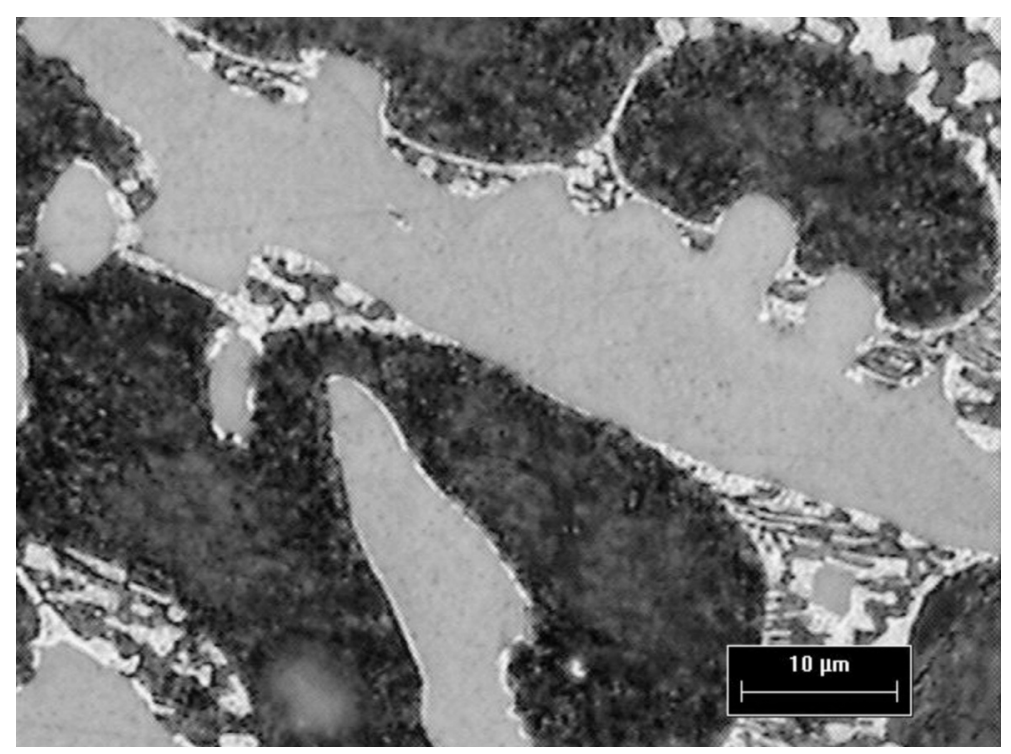

Figura 5. Aleación ACuZinc5, $\varepsilon$ - eutéctico. Velocidad de enfriamiento $18^{\circ} \mathrm{C} / \mathrm{s}$. $1.000 \mathrm{x}$.

Figure 5. ACuZinc5 alloy, $\varepsilon$-eutectic. Cooling rate $18^{\circ} \mathrm{C} / \mathrm{s}$. 1000x.

Es decir que, en caso de que inyectáramos la aleación directamente en un molde enfriado después de aplicar la condición de enfriamiento II, probablemente vamos a obtener una mayor fracción sólida de fase primaria, pero la microestructura seguirá siendo poco homogénea ya que adicionalmente a las particularidades del HPDC, partiríamos de una distribución y tamaños de fase primaria no uniformes ( Fig. 4 b)). Por la misma razón, si agitamos el baño metálico en estas condiciones, la microestructura final tampoco podría ser homogénea, pues fragmentaríamos dendritas de tamaño diverso. Adicionalmente se podrían formar rosetas con un tamaño mayor a $50 \mu \mathrm{m}$ pues se fragmentarían dendritas con un ancho próximo a esta medida antes de la maduración (Fig. 4 b)). Según trabajos anteriores un tamaño adecuado es entre 30 y $50 \mu \mathrm{m}{ }^{[21]}$.

En una patente precedente aplicaron agitación mecánica y ultrasónica en procesos SSM utilizando como referencia las curvas de enfriamiento de una aleación de Al AC4C, norma JIS [21]. En esta patente utilizaron puntos de la curva de enfriamiento antes, durante y después de la reacción primaria, pero no se expone bajo qué criterios se seleccionaron estas temperaturas. Desde el punto de vista microestructural, en esta patente, los mejores resultados se obtuvieron a una temperatura ligeramente inferior a la temperatura de líquidus. En esta patente se sugiere que el método puede ser utilizado, entre otras, en aleaciones de zinc sin tener en cuenta que la cinética de nucleación y crecimiento de las aleaciones de alu- munio y de zinc son diferentes. Por otro lado, la fracción sólida de la fase primaria es determinante al aplicar procesos SSM por su efecto sobre la viscosidad y se debe determinar un valor efectivo por tipo de aleación $^{[6]}$. En la patente mencionada, el autor indica que la fracción sólida a inicios de la solidificación debe ser mayor al 0,3 [21]. Sin embargo, en las aleaciones de zinc el porcentaje de fase $\varepsilon$ primaria depende además de la velocidad de enfriamiento (tabla II), del contenido de cobre entre otros factores $^{[7 \text { y } 13]}$. No obstante a estas diferencias hay una similitud con nuestros resultados. La microestructura mostrada en la figura 4 a) resultante de las condiciones de enfriamiento ensayadas es la que muestra una microestructura más uniforme y ésta se obtiene a temperaturas próximas a líquidus. Tentativamente, pudiera considerarse iniciar a partir de este punto de la curva de enfriamiento alguno de los procedimientos existentes para la obtención de una microestructura adecuada mediante procesos SSM, pero se partiría de una fracción sólida menor al 0,2 (tabla II). En consecuencia se requiere incrementar la fracción sólida pero conservando la uniformidad en tamaño y distribución de la fase primaria que se forma durante la nucleación y crecimiento antes de la maduración dendrítica (Fig. 4 a)). Hipotéticamente esto se podría lograr de dos formas:

- Con mantenimiento isotérmico, agitando el baño metálico desde su inicio a una temperatura entre el FRP y el PCD. 
- Aplicando agitación desde la temperatura de IRD con un enfriamiento lento dentro del rango de temperaturas de maduración de $\varepsilon$. La fragmentación de las dendritas primarias antes de que inicie o durante su maduración podría generar una mayor fracción de $\varepsilon$, con un tamaño y distribución más uniforme que el obtenido mediante procesos HPDC. Adicionalmente el incremento de fase primaria de menor tamaño implica un mayor substrato de nucleación para la fase $\eta$. Actualmente se lleva a cabo un procedimiento experimental para comprobar la hipótesis planteada.

\section{CONCLUSIONES}

- La aleación ACuZinc $5^{\mathrm{TM}}$ presenta una microestructura de dendritas finas de fase $\varepsilon$ primaria uniformemente distribuidas hasta que inicia la maduración o engrosamiento dendrítico. A partir de este punto, la morfología, tamaño, distribución y fracción sólida de la fase $\varepsilon$ primaria dependen de las condiciones de enfriamiento.

- La permanencia isotérmica a temperaturas próximas al inicio de la maduración de las dendritas primarias produce un incremento de la fracción de esta fase pero con tamaño y distribución no uniformes.

- Hipotéticamente, la obtención de una fracción, tamaño, morfología y distribución adecuados de la fase $\varepsilon$ primaria en la microestructura final podría obtenerse agitando el baño metálico antes del inicio de la maduración o engrosamiento dendrítico.

\section{REFERENCIAS}

[1] M.S. Rashid y M.D. Hanna, US Patent № 4.990, 310 (1991).

[2] M.D. Hanna y M.S. Rashid, US Patent № 5.603, 552 (1997).
[3] H.I. Laukli, C.M. Gourlay y A.K. Dahle, Metall. Mater. Trans. A 36 (2005) 805.

[4] Z. Zhen, Ma Qian, S. Ji y Z. Fan, Scripta Mater. 54 (2006) 207-211.

[5] M.C. Flemings, Metall. Mater. Trans. A 22 (1991) 952-981.

[6] O. Lashkari y R. Ghomashchi, J. Mater. Proc. Technol. 182 (2007) 229-240.

[7] D. Ma, Y. Li, S. C. NG y H. Jones. Acta Mater. 48 (2000) 1.741-1.751.

[8] L. Bäckerud, E. Krol y J. Tamminen, Solidification Characteristics of Aluminum Alloys, AFS/ Skanaluminium, Oslo; Norway, 1986, pp. 63 74.

[9] L. Bäckerud, G. Chai y J. Tamminen, Solidification Characteristics of Aluminum Alloys Vol. 2, AFS/Skanaluminium, Sweden, 1990, pp. 95-101.

[10] L. Arnberg, L. Bäckerud y G. Chai, Solidification Characteristics of Aluminum Alloys Vol. 3 Dendrite Coherency, AFS, Illinois, 1996.

[11] L.M. Veldman, A. Dahle, D. H. StJohn y L. Arnberg, Metall. Mater. Trans. A 32 (2001) 147.

[12] J. Oetinger, Die Casting Engineer, Julio (2002) $70-71$.

[13] E.D. Jareño, S.I. Maldonado y F.A. Hernández, J. Alloys Compd. 490 (2010) 524-530.

[14] J. Haughton y K. Bingham, Proceedings of the Royal Society of London 99A, 1921, pp. 47-68.

[15] G. Ghosh y J. Humbeeck, Aluminium-CopperZinc, Ternary Alloys, V.C.H., Weinheim, Vol. 5, 1992, pp. 92-112.

[16] S. Murphy, Zeitschrift fuer Metallkunde, 71 (1980) 96-102.

[17] H. Liang y Y.A. Chang, Journal of Phase Equilibria 19 (1998) 25-37.

[18] B.J. Li y C.G. Chao, Metall. Mater. Trans. A 30 (1999) 917-923.

[19] W. Kurz, Fundamentals of Solidification, Fourth Edition, Trans. Tech Publications, 1998, p. 111.

[20] D.M. Stefanescu, Science and Engineering of Casting Solidification, Second Edition, Springer, 2009, pp. 247-257.

[21] S. Aoyama, C. Liu, T. Sakazawa y Y. Pan, US Patent 7,051,784 B2 (2006). 